\title{
UbiKit: Learning to Prototype for Tangible and Ubiquitous Computing
}

\author{
Florian Güldenpfennig ${ }^{1}$, Francisco Nunes ${ }^{1,2}$, Özge Subasi ${ }^{1}$, Michael Urbanek ${ }^{1}$ \\ ${ }^{1} \mathrm{HCl}$ Group, TU Wien, Argentinierstraße 8, Vienna, Austria \\ ${ }^{2}$ Fraunhofer Portugal AICOS, Rua Alfredo Allen 455, Porto, Portugal \\ \{florian.gueldenpfennig, francisco.nunes, oezge.subasi, michael.urbanek\}@tuwien.ac.at
}

\begin{abstract}
As more and more ubiquitous computing (ubicomp) technologies pervade our daily lives, it is becoming increasingly important to train interaction design students to create these technologies. However, teaching students to design ubicomp technologies can be challenging, because creating a prototype, which people can use and students can learn from, usually requires a long setup and development phase. While there are some prototyping kits available, such as Arduino or the Raspberry $\mathrm{Pi}$, they often require relatively steep learning curves before anything sophisticated can be prototyped. For this reason, we created UbiKit, a quick-to-setup, reusable, and affordable toolkit for the rapid design of ubicomp technologies that enable people to co-explore different scenarios and focus on the users. We piloted UbiKit in a Master-level course on ubicomp related topics and reflect on its potential to help streamline the prototyping and overall learning process for students being trained in our research area.
\end{abstract}

Tangible Prototyping Kit. Prototyping. Ubicomp. Interaction Design Education.

\section{INTRODUCTION}

Designing interactive products, like ubiquitous computing (ubicomp) technologies, is a complex challenge. Designers have to consider different needs and contexts of users, the technological options and constraints, as well as possible interdependencies (Sellen, Rogers, Harper, \& Rodden 2009). Consequently, to become competent practitioners, students in interaction design need to acquire a diverse set of skills spanning technological know-how, competencies in design, and an ability to investigate the life of their potential users.

Learning to design for ubicomp scenarios is increasingly important as these artefacts become part of the fabric of our everyday lives. However, it is often hard to teach ubicomp related topics due to the effort it takes to reach a sophisticated prototype with which students can experiment and use to learn from. Many applications involve physical and interactive artifacts that go beyond conventional desktop computing. Sensors, actuators, and embedded microcontrollers introduce rich opportunities for interaction, but also complexity that was unknown to traditional (desktop) software development or product design (Obrenović 2012). In fact, it is common for the setup and development of ubicomp technologies to take several days or weeks, even when using a physical prototyping kit such as Arduino, Gadgeteer or Raspberry Pi. The numerous components and pieces of ubicomp take time to master and, by the time students understand them, there is possibly not much time left in the course to learn about people's needs or to reflect about potential uses or even pitfalls of their designs.

People can only learn what they experience or, better said, experienced (Vygotsky 1978). If students only focus on the technical setup and development of ubicomp technologies, it is realistic to hypothesize that they will not become familiar with the humanistic and design perspectives of designing ubicomp technologies. For being able to learn about non-technical facets of ubicomp system design, students need to dedicate time to those activities, which is not possible if students develop a prototype from scratch.
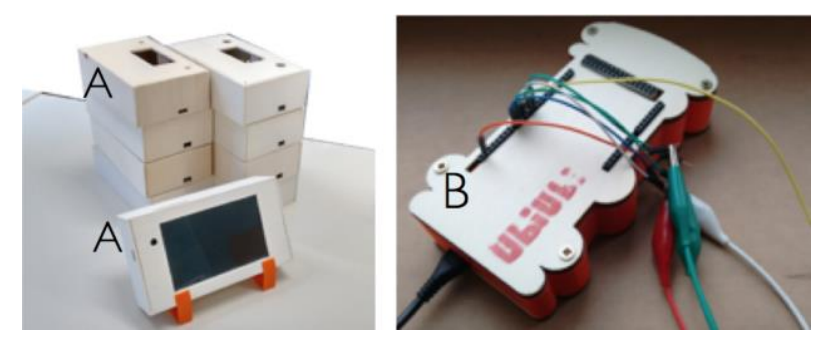

Figure 1: The UbiKit consisting of UbiScreen $(A)$ and UbiUbi (B). Note: A shows multiple UbiScreens. 

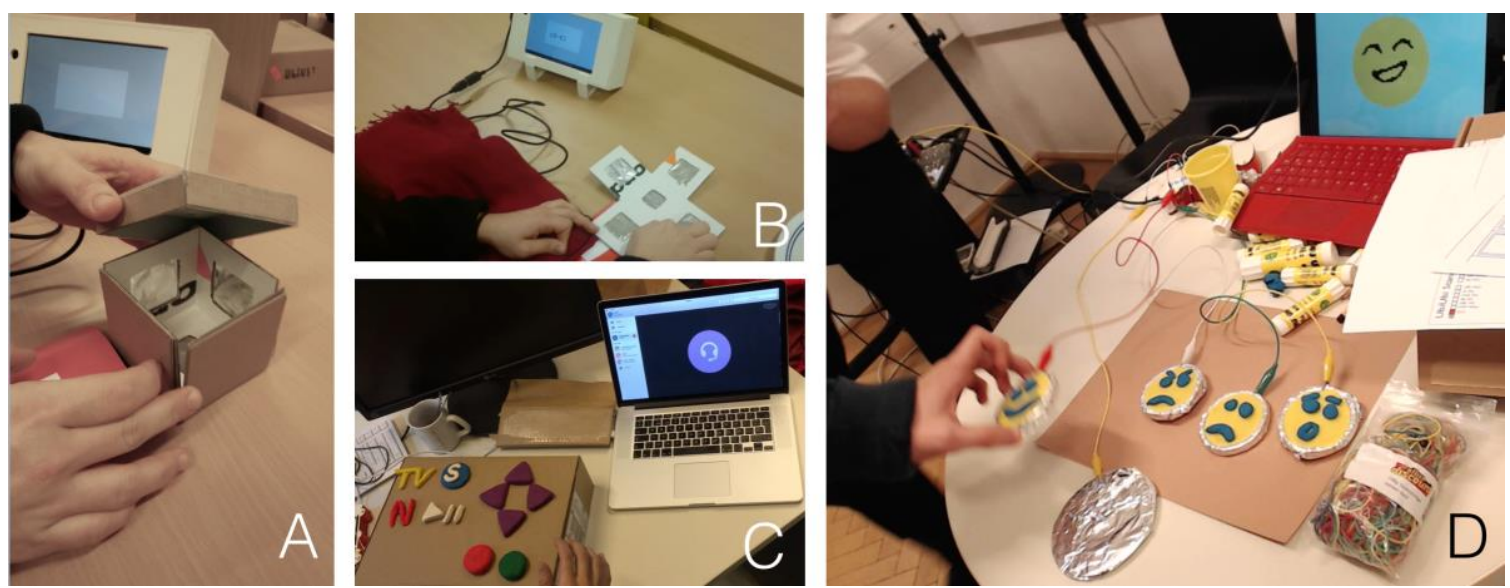

Figure 2: Different tangible interaction design concepts explored by students using UbiKit. Aluminum foil connected to the UbiUbi and presented as an expandable cube as a game controller for the UbiScreen ( $A-B$; note, the UbiUbi is covered with a blanked). Alternative and accessible interfaces for computers prototyped with Play-doh and the UbiUbi (C-D).

Moreover, the lack of training in the humanistic and design activities can be problematic in an area that prides itself on being interdisciplinary. Similar to others (Churchill, Bowser, \& Preece 2013), we believe that Human-Computer Interaction $(\mathrm{HCl})$ education should train students to become proficient in different areas of practice that coexist in $\mathrm{HCl}$, and that is only possible if technical aspects are not the sole focus of education.

In this paper we present UbiKit, a prototyping kit that bootstraps the setup and development of ubicomp technologies. UbiKit (illustrated in Figure 1) had two primary requirements. First, the kit had to be appropriate to rapidly create sophisticated prototypes, so students could spend time on exploring design options and investigating the context of use, rather than 'only' on setting up or developing the system. Second, it had to be versatile to multiple ideas, reusable, sustainable, and low-cost given that teaching budget can be quite limited.

In the remainder of this paper, we will describe the concept and functionalities of UbiKit in more detail. We will also provide a brief overview of the related work that inspired our toolkit. Subsequently, we will report and discuss first experiences and observations from using UbiKit in a Master-level interaction design course involving 27 students. Finally, we will detail our next steps to advance education in $\mathrm{HCl}$ and interaction design.

\section{UBIKIT = UBISCREEN + UBIUBI}

As motivated in the introduction, in our interaction design course, we made the creation of physical prototypes the centerpiece of the learning experience, and we designed a number of exercises to help students reach a high level of internal motivation for building up competencies through making prototypes. However, as we had restricted resources we needed to make use of affordable and reusable materials. Since we couldn't identify a suitable product on the market, we created the UbiKit. This modular system consisted of (see Figure 1):

- a main computing module featuring an Android tablet built into a wooden casing (the UbiScreen) and

- additional components like a generic keyboard/mouse emulator (the UbiUbi) to be easily connected to the main computing module via USB cable.

The UbiKit acted like a blank canvas for interaction design and was to be extended and modified by the students both programmatically and physically for designing tangible and ubicomp interactions. For example, students were able to connect conductive objects to the UbiUbi to make up and to experience innovative user input modalities (for examples, see Figure 2 and 3 ). Students could also create additional hardware (mock-ups) or software applications to be integrated with the UbiKit system (examples are shown in Figure 4 and 5). If required, the UbiScreen could be replaced by other Android devices (for example, to try out a different form factor).

The rationale of UbiKit was to provide students with a ubicomp educational toolkit to be taken home, which is timely, extendable, affordable, and accessible to students with different levels of skill (Figure 1 left side shows some kits to be provided to the students; all components can be stored in the back of the UbiScreen box). As outlined in the introduction, it was of particular importance that 
UbiKit would afford the creation of more sophisticated prototypes while at the same time providing enough space for creative explorations by removing technical barriers or constraints (i.e., the students should be supported to focus on design or design thinking, not challenges in technical implementations which do not relate directly to the current problem space).

Figure 2 displays some exemplary work done by interaction design students. UbiKit facilitated the quick implementation of a game controller featuring touch sensors made of aluminium foil $(A-B)$, accessible controls for basic functions like Internet telephony or television (C), and a 'mood interface' (D). In those examples, the students spent less time on the implementation, and more on trying out different ideas.

For the projects displayed in Figure 4 and 5, the students had two weeks to create each prototype.

\section{RELATED WORK}

There are many exciting tools like the Arduino (Arduino 2016), Raspberry Pi (Raspberry Pi 2016) or Makey Makey (Collective \& Shaw 2012) that relate to the UbiKit. Each one of these tools comes with its own strengths and audiences. For example, the Arduino is a microcontroller board including an IDE for programming sensors and actors, the Raspberry $\mathrm{Pi}$ constitutes a powerful single-board computer, and the Makey Makey is a playful mouse/keyboard emulator. The UbiKit seeks to combine the individual advantages of these

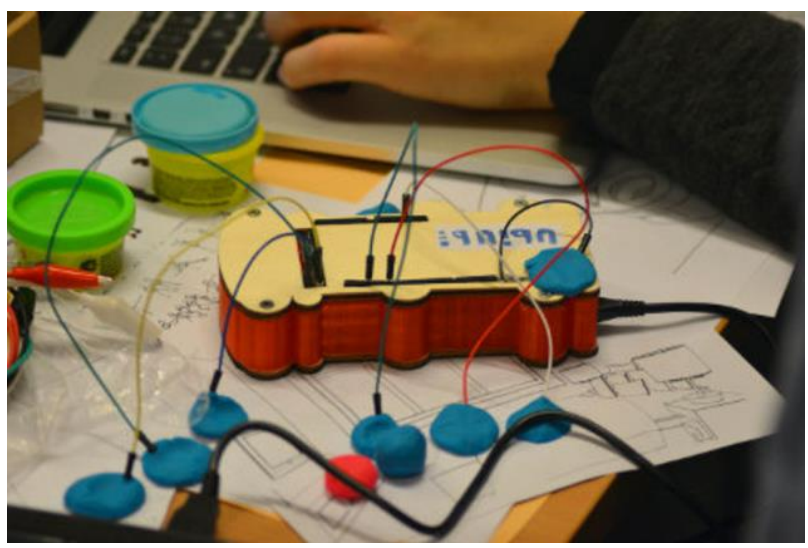

Figure 3: UbiUbi during a design workshop. Play-Doh (or any other conductive objects like e.g. aluminum foil) connected to wires acted as touch sensors and allowed the easy setup of different user input keys. Technically, the UbiUbi is an extension of the Makey Makey

(Collective \& Shaw 2012).

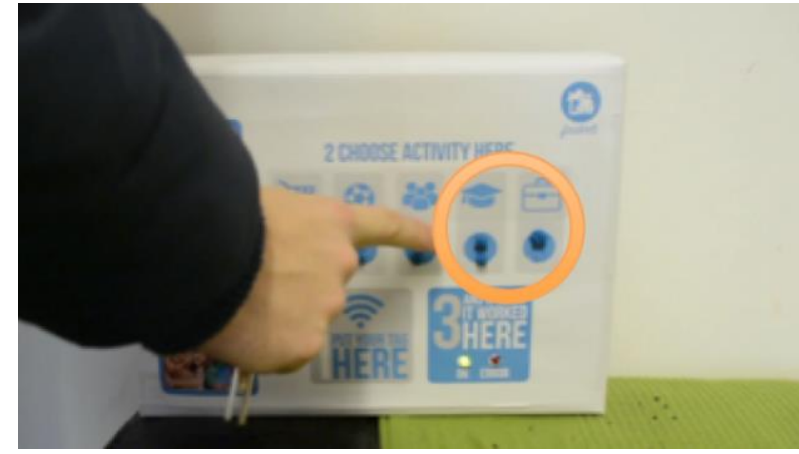

Figure 4: Tangible message board "Finderly" designed by a group of students for tracking family's whereabouts. Here, the main computing module was covered with a paper printout to prototype a smart device for organizing family activities. Touch interfaces were prototyped using UbiUbi (hidden behind the printout/cardboard) with some blue PlayDoh (see circle).

products in one toolkit. For example, the UbiUbi (see Figure 3) can be regarded as a clone of the Makey Makey with extended functionalities (it supports more keys to be attached). In combination with UbiScreen, UbiUbi can reveal its full potential when easy-to-learn and freely-available programming languages like Processing (Processing 2016) are used to trigger actions on the screen. Figure 4, for example, displays a number of touch keys that were prototyped using the UbiUbi. Here, the main computing device (for processing the UbiUbi input) was covered by some paper printouts by students to mockup a fully functional tangible device.

From an educational perspective, in our overall approach, we were inspired by the work of others, who were considering novel concepts for interaction design education. For example, Reimer and Douglas (2003) adapted the classical design studio approach to their $\mathrm{HCl}$ course. Obrenović (2012) introduced an educational framework for learning interactivity in computing. In principal, these researchers follow the same agenda as we do with UbiKit. The goal is to create situations where technology can be explored in a productive way, without technical issues and implementation challenges interfering with the actual learning experience.

In summary, we extend existing related work by recombining existing products and features into a novel toolkit optimized for our education needs as outlined in the introduction. Moreover, and of equal importance, we seek to study the implications for interaction design education, which is not part of the agenda of most electronics tinkering kits. 


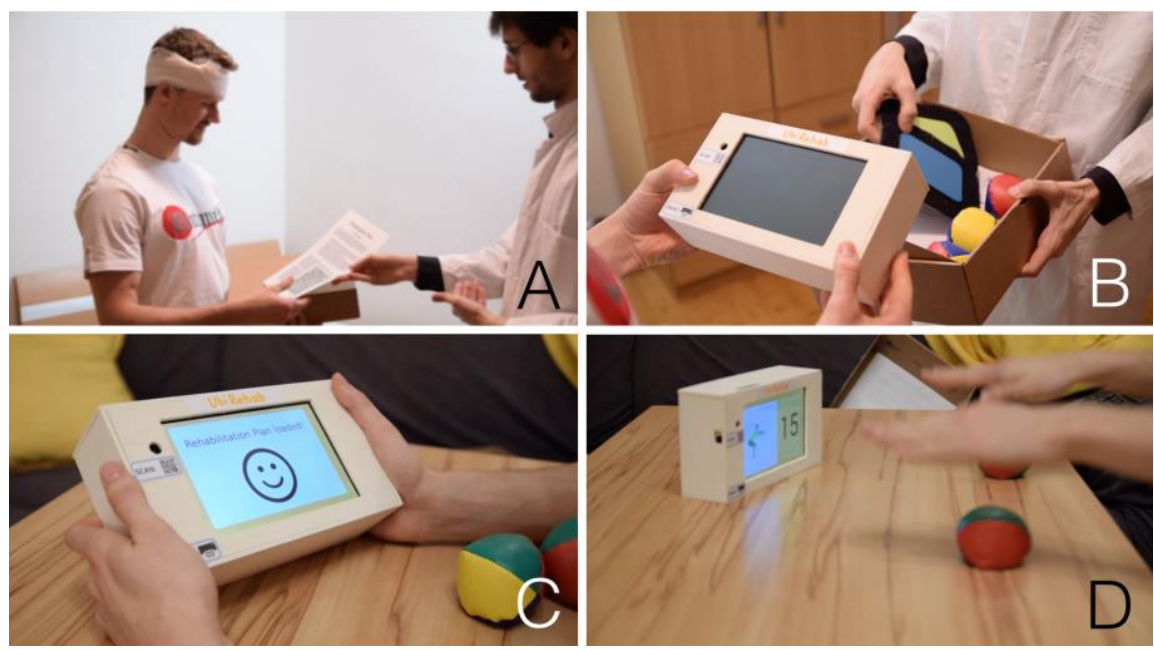

Figure 5: Film excerpts from a video prototype by the participants using UbiKit (UbiScreen) to illustrate their interaction design.

\section{RESEARCH APPROACH AND FIRST EXPERIENCES WITH UBIKIT}

In our research, we take a qualitative approach for analyzing the students' learning experiences and how they made use of the different toolkits we offered them for their constructions (both in a physical and cognitive sense; see discussion). Therefore, in our research we are interested in rich descriptions as provided by, for example, Thematic Analysis (Braun \& Clarke 2006) and not so much in the results of, say, controlled experiments in a Post-Positivist tradition.

At the moment, we are analyzing the prototypes created by the students, but we can already discuss our initial observations. In fact, the figures in this paper were taken from this course and the students gave their consent after the completion of the class, allowing us to show their ideas and photos. The prototypes presented in this paper were created during two exercises. The first exercise was conducted as part of an introductory design workshop where the students $(n=27$; three per group) were instructed to create alternative and tangible user interfaces to either the UbiScreen or a conventional computer. Work-time was restricted to four hours including one hour of introduction given by the first author of this paper (the photos in Figure 2D and 3 were captured during the workshop). Exercise 2 was "homework" where the students were given the task to design an ubicomp system for improving wellbeing or leisure time (see Figure 4 and 5).

We have not yet employed an in-depth analysis of the collected data for this paper. However, in the remainder of this section, we present preliminary themes that emerged from our observations regarding the UbiKit so far.

\section{Rapid prototyping of a broad variety of user input options and devices}

First of all, the UbiUbi proved to be a useful tool for the rapid prototyping of different touch input options. For example in Figure 2, the students used Play-Doh and aluminum foil to shape their own "buttons" and "switches". Traditionally, implementing physical buttons in a prototype required knowledge about electronics, the ability to solder, and non-trivial programming skills. The UbiUbi device however superseded these setup and development steps in the prototyping process by incorporating cheap and reusable materials such as Play-Doh. In Figure 4, we can see a number of conventional or rather simple switches which constituted the primarily interaction interface of a prototype for a family activity organizer ("Finderly"). On the touch of one of these switches, this user input was interpreted by the computing module (not visible in the image) and the devices adjusted the whereabouts of family members accordingly.

Other students (see Figure 2) came up with unique game controllers or accessible controls in the shape of extra large buttons with a clean layout. Figure $2 \mathrm{~A}$ and $\mathrm{B}$, for example, shows a foldable cube, which could be expanded to a cross-shaped user interface featuring aluminum touch panels. This kind of "three-dimensional" and at the same time "flat" design was facilitated in particular by the affordances of the UbiKit.

Lo-fi prototyping and triggering of screen mock-ups The students were able to make good use of the UbiKit by implementing simple applications in Processing that triggered different mock-up 
screens on the touch of different UbiUbi "buttons". This kind of use facilitated the rapid creation of interactive prototypes where the UbiScreen reacted to user input by displaying corresponding information. While their prototypes were seemingly interactive, the students did not have to invest much effort to accomplish this behavior.

\section{UbiKit and video-prototyping}

As evident in Figure 4 and 5, the UbiKit was augmented by the students with additional information (e.g., paper printouts of designs and cardboards) and props (additional peripheral devices like "smart" pads or balls). By these means, they were able to create appealing and powerful demos and also to frame them as video prototypes for tangible and ubiquitous technology. Figure 5, for example, displays the work of students where they embedded the UbiKit into a set of (smart) health utensils. Their video prototype explained their concept convincingly, namely, that they envisioned a product for rehabilitation that is prescribed by the doctor and used by the patient at home. This patient is supported in exercising by smart utensils from the kit such as exercise pads, video instructions, exercise log, and so on. Thus, we found that UbiKit was particular powerful in combination with the video-prototyping technique (Buxton 2007).

\section{Ease of use, sophistication, and skills required}

One of the strengths of UbiKit in our preliminary study was its versatility. On the one hand, the students used it for a variety of different products and UbiKit could be used to implement prototypes of different fidelity. One the other hand, it could be used by students from different levels of experience and skillset. Depending on a prototype's intention and stage of the design process, it can also be appropriate to invest more or less effort in its implementation (Buxton 2007). Here, UbiKit seemed to be useful during different stages, in particular, in the lo-fi and mid-fi phase of prototyping. Nonetheless, if appropriate, students can also use the Android API to create more bespoke programs and make use of UbiKit's standard functions and devices such as Internet connectivity or a built-in camera, since UbiScreen is technically an Android tablet. As mentioned in section 2, the UbiScreen can also be replaced by a different Android device, for example, should a different form factor be required.

\section{Focus on the user in the design of ubicomp}

The observations made in the paragraph above relate to the occurrence of "truly" user-centered design. By this we mean that in the prototypes (see Figures 4, 5), we found elaborated and thoughtful concepts instead of technical demonstrations and poorly motivated gadgets.
Since the technical setup and development was achieved in a short amount of time, students were quickly free to think about their users and the contexts where their technology would be used. Students focused more time on discussing the advantages and drawbacks of their solution, the characteristics of their users, and the problem they were solving, instead of the electronics and the software that was needed to power their idea. This was facilitated by the ease of use in UbiKit, which allowed the students to spend a good proportion of their time on investigating the user needs.

\section{DISCUSSION AND CONCLUSION}

With our research endeavor, we seek to contribute a qualitative account of the training of students in tangible ubicomp and interaction design with a people-centered perspective. We focus in particular on creating tangible and interactive prototypes, and aim to highlight how constructing and experiencing physical systems can facilitate the teaching goal of educating responsible and competent interaction designers. Our work takes a Constructivist stance, both in the approach to learning and research. It is our belief that the student or learner has to construct their own understanding and knowledge about technologies, social fabrics, contexts or the world as a whole. Interestingly, in our teaching this is accomplished by the physical activity of constructing prototypes, among other activities. It is our objective as the educators to create appropriate situations and contexts for the students to be successful in their understanding (Vygotsky 1978). To this end, we conceived UbiKit to remove technical barriers, constraints, and distractions.

\section{FUTURE WORK}

Our next steps in studying the UbiKit is to setup a user study for observing the system in the field in a more systematic way. To do so, we are currently finalizing the study plan. At the same time, we are discussing and complementing UbiKit with additional pieces of hardware. Similar to UbiUbi, this will be add-on components that can extend the kit with extra options for user input and output (e.g., a temperature sensor or buzzer). One important challenge though will be to identify the right kind of balance between functions provided and simplicity. It is desirable to allow the prototyping of a broad variety of tangible prototypes using UbiKit. However, we also want to keep the system sustainable, affordable, and clear in its conception. 


\section{REFERENCES}

Arduino. (2016). http://www.arduino.cc, accessed 15 May 2017.

Braun, V., \& Clarke, V. (2006). Using Thematic Analysis in Psychology. Qualitative Research in Psychology, 3(2), 77-101.

Buxton, B. (2007). Sketching User Experiences Getting the Design Right and the Right Design. Morgan Kaufmann, San Francisco, CA, USA.

Churchill, E. F., Bowser, A., \& Preece, J. (2013). Teaching and learning human-computer interaction: past, present, and future. interactions, 20(2), 44-53.

Collective, B. s. M., \& Shaw, D. (2012). Makey Makey: improvising tangible and naturebased user interfaces. Paper presented at the Proceedings of the Sixth International Conference on Tangible, Embedded and Embodied Interaction, Kingston, Ontario, Canada.

Obrenović, Ž. (2012). Rethinking HCl Education: Teaching Interactive Computing Concepts Based on the Experiential Learning Paradigm. interactions, 19(3), 66-70.

Processing. (2016). https://processing.org/ accessed 15 May 2017.

Raspberry Pi. (2016). www.raspberrypi.org/ accessed 15 May 2017.

Reimer, Y. J., \& Douglas, S. A. (2003). Teaching $\mathrm{HCl}$ Design With the Studio Approach. Computer Science Education, 13(3), 191205.

Sellen, A., Rogers, Y., Harper, R., \& Rodden, T. (2009). Reflecting human values in the digital age. Commun. ACM, 52(3), 58-66.

Vygotsky, L. S. (1978). Mind in society: Development of higher psychological processes. Harvard University Press, Cambridge. 